\title{
Mode Selection and Tuning Mechanisms in Coupled-Cavity Terahertz Quantum Cascade Lasers
}

\author{
Xiaoqiong Qi, Iman Kundu, Paul Dean, Gary Agnew, Member, IEEE, Thomas Taimre, Alexander Valavanis, \\ Andrew T. Grier, Edmund H. Linfield, Alexander Giles Davies, Dragan Indjin, \\ and Aleksandar D. Rakić, Senior Member, IEEE
}

\begin{abstract}
We present a model for longitudinal mode competition in coupled-cavity $(\mathrm{CC})$ terahertz $(\mathrm{THz})$ quantum cascade lasers (QCLs) by using a scattering matrix method and multimode reduced rate equations ( $R R E s)$. The dependence of the mode selection and tuning characteristics on various device parameters is systematically investigated, including the net waveguide loss, the optical length of the passive cavity, and the heat-sink temperature for different relationship between the active and passive cavity lengths. The changes in eigenmode frequencies due to variations of device parameter are calculated before solving the RREs. The mode selection and tuning results obtained from solving the nonlinear RREs could be well explained by the linear scattering matrix analysis. The mode tuning process simulated by the proposed model is compared with experimentally measured data, yielding good agreement. Comprehensive study of the influence of the key device parameters on the performance of CC THz QCLs provides potential design rules for single-mode operation with either wide frequency tunability or high stability.
\end{abstract}

Index Terms-Coupled-cavity, mode tuning, quantum cascade lasers (QCL), terahertz (THz).

\section{INTRODUCTION}

$\mathbf{T}$ HE terahertz (THz) region of the electromagnetic spectrum (frequency: $0.5-10 \mathrm{THz}$, wavelength: $600-30 \mu \mathrm{m}$,

Manuscript received October 7, 2016; revised February 7, 2017; accepted February 9, 2017. This work was supported in part by the Australian Research Council Discovery Project (DP160103910); in part by the Engineering and Physical Sciences Research Council, UK (COTS Program EP/J017671/1); in part by the Royal Society Wolfson Research Merit Award scheme (EHL, WM150029; AGD, WM110032); and in part by the European Cooperation in Science and Technology (BM1205 and MP1406 Actions).

X. Qi, G. Agnew, and A. D. Rakić are with the School of Information Technology and Electrical Engineering, The University of Queensland, Brisbane, Qld. 4072, Australia (e-mail: x.qi1@uq.edu.au; gary.agnew@uq.net.au; rakic@itee.uq.edu.au).

I. Kundu, P. Dean, A. Valavanis, E. H. Linfield, A. G. Davies, and D. Indjin are with the School of Electronic and Electrical Engineering, University of Leeds, Leeds LS2 9JT, U.K. (e-mail: I.Kundu@leeds.ac.uk; P.Dean@leeds.ac.uk; a.valavanis@leeds.ac.uk; e.h.linfield@leeds.ac.uk; G.Davies@leeds.ac.uk; d.indjin@leeds.ac.uk).

T. Taimre is with the School of Mathematics and Physics and School of Information Technology and Electrical Engineering, The University of Queensland, Brisbane, Qld. 4072, Australia (e-mail: t.taimre@uq.edu.au).

A. T. Grier is with Seagate Technology, Londonderry BT48 OLY, U.K. (e-mail: el09a2g@leeds.ac.uk).

Color versions of one or more of the figures in this paper are available online at http://ieeexplore.iee.org.

Digital Object Identifier 10.1109/JSTQE.2017.2668600 photon energy: 2-40 meV) has attracted considerable attention recently for its potential applications in chemical sensing, non-invasive imaging and spectroscopy [1]-[3]. THz quantum cascade lasers (QCLs) were first successfully demonstrated in 2002 [3]. Over the last decade, THz QCL technology has witnessed rapid development, and THz QCLs can now emit output powers $>1 \mathrm{~W}$ [4], within the 1.2-5.4 THz range [5]-[7], and operate at temperatures of up to $\sim 200 \mathrm{~K}$ in pulsed mode [8]. Single-mode operation THz QCLs with wide frequency tuning range are particularly required for sensing, spectroscopic, and imaging applications [9], [10]. However, the intrinsic frequency tuning range of single cavity QCLs by adjustment of the injection current and temperature are limited to approximately $0.05 \mathrm{~cm}^{-1}$ and $0.1 \mathrm{~cm}^{-1}$, respectively [11], [12]. A variety of tuning techniques for $\mathrm{THz}$ QCLs have been reported. Large tuning range $(>20 \mathrm{GHz}) \mathrm{THz}$ emission have been achieved by applying external grating, MEMS, or heterogeneous active region in QCLs [13]-[15], but suffer from slow tuning, operation difficulties or device complexity.

Coupled-cavity (CC) THz QCLs offer fast frequency tuning as a function of localized electrical heating of an optically coupled passive cavity [16]-[18]. The spectral function of coupled-cavity GaAs/AlGaAs THz QCLs has been calculated using transfer (T)-matrix method [16]. However, the mode modulation depth predicted by T-matrix approach $(12 \%)$ is much smaller than the measured $(62 \%)$ due to the absence of influence of laser action in active cavity. It was also concluded that the interplay between the photon density and electron population of the intersubband laser levels has to be included to accurately describe the experimentally observed switching of the mode structure. In addition, single-mode operation with excellent side mode suppression ratio (SMSR) above $34 \mathrm{~dB}$ was obtained in [17], where the T-matrix approach accurately predicted the eigenmode frequencies. However, the most important property of the CC QCLs, namely, mode selection and frequency tunability and their dependence on the key device parameters have not been systematically investigated. Discrete Vernier frequency tuning of CC THz QCLs was analyzed in [18], where the gain spectrum of the QCL was not taken into account in the mode hopping simulations. All reported theoretical analyses of CC THz QCLs have used only the transfer matrix method, which can only predict mode-selective filtering characteristics 


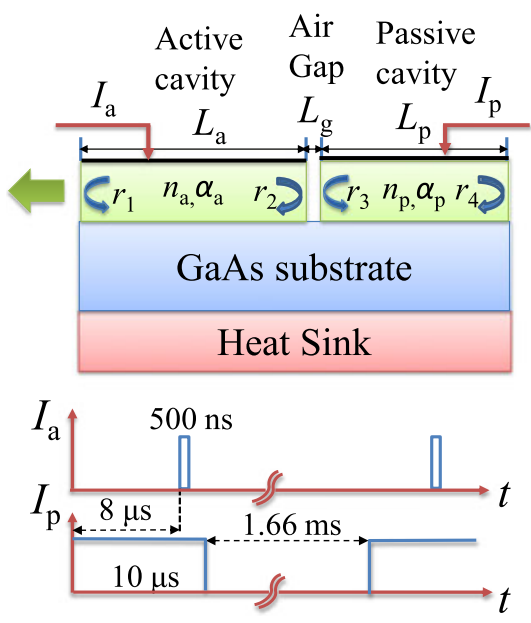

Fig. 1. Schematic diagram of the coupled-cavity multimode quantum cascade laser configuration.

below threshold. In this work, we combined the scattering matrix method and multi-mode reduced rate equations (RREs) analysis coupled with the full RE Schrödinger-Poisson (S-P) energy balance electron scattering transport calculations [19], to accurately model the mode selection and tuning characteristics in CC THz QCLs. The scattering matrix method is used to calculate the eigenmode frequencies and threshold gain, while the multi-mode RREs coupled with S-P simulation describe the laser behavior above the threshold, especially the influence of the gain spectrum. Systematical investigation of the dependences of the mode selection and frequency tunability on key device parameters in CC THz QCLs were conducted for different relationships between the active cavity length $\left(L_{\mathrm{a}}\right)$ and the passive cavity length $\left(L_{\mathrm{p}}\right)$, see Fig. 1 , i.e $L_{\mathrm{a}}>L_{\mathrm{p}}, L_{\mathrm{a}} \sim L_{\mathrm{p}}$ and $L_{\mathrm{a}}<L_{\mathrm{p}}$. An analog approach was applied to analyze and design CC diode lasers by Coldren et al. [20]-[22]. In spite of the recognized importance of the mode selection and frequency tuning in CC THz QCLs, no related theoretical investigation has yet been reported.

The purpose of this paper is threefold. Firstly, to generalize the RRE model from single-mode THz QCLs [19] to multimode THz QCLs, so that the interplay between the multi-mode photon densities and the electron densities of the intersubband laser levels could be accurately described. Secondly, to evolve the model for CC QCLs based on the scattering matrix method and integrate it with the multi-mode RREs. This allows us to formulate design rules for CC THz QCLs based on our observations of how mode selection and tuning characteristics depend on the key device parameters. The spectral power density (SPD) distribution of the eigenmodes and their dependence on the net waveguide loss and the effective refractive index of the passive cavity, and the heat sink temperature of the $\mathrm{CC}$ device are investigated. Finally, the proposed model contains eigenmode calculations for CC THz QCLs, on which the multi-mode RREs are based, so that the continuous frequency tuning of the dominant mode could also be modelled.

Fundamentally, the CC structure leads to a frequencydependent threshold gain. The net waveguide loss and effective refractive index of the passive cavity determine the modulation depth and phase of the threshold gain, respectively, which combines with the gain spectrum determines the dominant mode and the SMSR. Furthermore, it was found that eigenmode distributions on the threshold gain spectrum play an important role in mode tunability of CC THz QCLs especially when $L_{\mathrm{p}} \geq L_{\mathrm{a}}$. Various locations of eigenmodes (at peaks, rising and falling edges) can enhance the mode tunability. Technically, by varying the passive cavity refractive index over a wide range and observing the effect this has on mode and frequency tunability, we are able to choose a passive cavity length or drive current that provides a suitable mode tunability. Likewise, by exploring the heatsink temperature over a wide range, we are able to choose a temperature that provides the desired combination of mode and continuous frequency tunability. The proposed modelling approach has been validated by experimentally observed mode tuning as a function of heating power applied to the passive cavity.

The article is organized as follows. Section II describes the scattering matrix based linear coupled-resonator analysis used to calculate the threshold gain and eigenmode frequencies. This is followed by multi-mode RRE analysis for mode selection characteristics, in Section III. The results and discussion of the effects of device parameters on mode selection and frequency tuning are given in Section IV. Section V compares the simulation results with experimental data. Finally, Section VI presents our conclusion.

\section{LINEAR COUPLED-RESONATOR ANALYSIS}

The exemplar device selected for modelling is a $150-\mu \mathrm{m}$-wide and 11.6- $\mu \mathrm{m}$-thick THz CC QCL with a bound-to-continuum active region based on $\mathrm{GaAs} / \mathrm{Al}_{0.15} \mathrm{Ga}_{0.85}$ As material system, as described in [23]. The key parameters in this two-section CC THz QCL are shown in Fig. 1, where the active cavity of length $L_{\mathrm{a}}$ and the passive cavity of length $L_{\mathrm{p}}$ are separated by a narrow air gap with length of $L_{\mathrm{g}}$. We focus on a device with an active cavity length $L_{\mathrm{a}}=1.5 \mathrm{~mm}$ and an air gap length $L_{\mathrm{g}}=13 \mu \mathrm{m}$, which are the dimensions of a fabricated device we used to experimentally verify our theoretical model. To simulate three different geometries of the device $\left(L_{\mathrm{a}}>L_{\mathrm{p}}, L_{\mathrm{a}} \sim L_{\mathrm{p}}\right.$, and $L_{\mathrm{a}}<$ $L_{\mathrm{p}}$ ), passive cavity lengths $L_{\mathrm{p}}$ of $0.25 \mathrm{~mm}, 1.4 \mathrm{~mm}$, and $3.4 \mathrm{~mm}$ were used. The operational condition of the CC THz QCL device simulated in this article is the same as described in [18] where the active cavity is driven by 500 ns-long pulses, which is triggered by a $600 \mathrm{~Hz}$ reference frequency and the passive cavity is heated using a train of $10-\mu$ s-long current pulses at a repetition rate of $8.21 \mathrm{kHz}$, gated by the same $600 \mathrm{~Hz}$ reference, as shown in Fig. 1. In this scheme, the amplitude of the active cavity current pulses is fixed at $0.75 \mathrm{~mA}$ (above the lasing threshold) and the amplitude of the passive cavity pulses vary below its threshold to control the dominant longitudinal mode of the device. We can neglect thermal crosstalk between the active and passive cavities due to the narrow pulses applied on both cavities.

When the passive cavity is operated below threshold or well above optical power rollover point, it can be assumed to behave as a linear equivalent mirror [20]. Based on complex scattering transmission matrix theory [22], the air gap and passive cavity can be described by an effective complex mirror. This way 
the CC QCL can be described as a single cavity (SC) with an effective complex mirror.

The net transfer function of the CC QCLs can be described by $[20]$ :

$$
H(\nu)=\frac{t_{a} t_{p} S_{s 21} \sqrt{\left(1-r_{4}^{2}\right)\left(1-r_{1}^{2}\right)}}{1-\left(S_{s 12}^{2}-S_{s 11}^{2}\right) r_{1} r_{4} t_{a}^{2} t_{p}^{2}-S_{s 11} r_{4} t_{p}^{2}-S_{s 22} r_{1} t_{a}^{2}}
$$

The parameters in (1) are defined in Table I. The eigenmode frequencies of the CC QCL, $\nu_{m}$, are determined by the frequencies where the transfer function has local maxima or zeros of the denominator of $H(\nu)$ [20]. The linear coupled-resonator analysis of the coupled cavity above is essential for accurate frequency tuning analysis in CC QCLs. Firstly, the CC eigenmode calculation and updating with the varying device parameters (the net waveguide loss, the optical cavity lengths and heat sink temperatures) are necessary for frequency tunability study. Secondly, the threshold gain experienced by the $\mathrm{CC}$ eigenmodes can be selectively lowered by an appropriate selection of the device parameters. The threshold gains at the eigenmodes combine with the gain spectrum to predict the potential dominant mode, which is nearest to the net gain maximum above threshold.

\section{Mode Competition Analysis Through Reduced RATE EQUATION AND THERMAL EQUATION}

Mode competitions in CC THz QCLs can be described by multi-mode reduced rate equations for each longitudinal eigenmode frequency, calculated from the linear coupled resonator analysis. The multi-mode rate equations are a modification of those for single-mode QCLs [19] based on the classical QCL model as shown below:

$$
\begin{aligned}
\frac{d N_{3}(t)}{d t}= & \frac{\eta_{3} I_{\mathrm{a}}(t)}{q}-\left(N_{3}(t)-N_{2}(t)\right) \sum_{m=1}^{N} G_{m} S_{m}(t) \\
& -\frac{N_{3}(t)}{\tau_{3}} \\
\frac{d N_{2}(t)}{d t}= & \frac{\eta_{2} I_{\mathrm{a}}(t)}{q}+\left(N_{3}(t)-N_{2}(t)\right) \sum_{m=1}^{N} G_{m} S_{m}(t) \\
& +\frac{N_{3}(t)}{\tau_{32}}+\frac{N_{3}(t)}{\tau_{\mathrm{sp}}}-\frac{N_{2}(t)}{\tau_{2}} \\
\frac{d S_{m}(t)}{d t}= & M\left(N_{3}(t)-N_{2}(t)\right) G_{m} S_{m}(t)-\frac{S_{m}(t)}{\tau_{\mathrm{p} m}} \\
& +\frac{M \beta_{\mathrm{sp}} N_{3}(t)}{\tau_{\mathrm{sp}}} \\
\frac{d T_{\mathrm{p}}(t)}{d t}= & \frac{1}{m_{\mathrm{p}} c_{\mathrm{p}}}\left[I_{\mathrm{p}}(t) V_{\mathrm{p}}(t)-\frac{T_{\mathrm{p}}(t)-T_{0}}{R_{\mathrm{th}}}\right]
\end{aligned}
$$

\begin{tabular}{|c|c|c|}
\hline Symbol & Description & Value / Definitions \\
\hline$r_{\text {eff }}$ & $\begin{array}{l}\text { Reflection coefficient of the } \\
\text { effective mirror }\end{array}$ & $\begin{array}{c}r_{\mathrm{eff}}= \\
S_{S 11}[1+\sigma \tilde{R} /(1-\tilde{R})]\end{array}$ \\
\hline$\sigma$ & Gap factor & $\begin{array}{c}\sigma= \\
S_{\mathrm{s} 21} S_{\mathrm{s} 12} / S_{\mathrm{s} 11} S_{\mathrm{s} 22}\end{array}$ \\
\hline$\tilde{R}$ & Resonance of $r_{\mathrm{eff}}$ & $\begin{array}{c}\tilde{R}= \\
S_{s 22} r_{3} \exp \left(-2 j \tilde{\beta}_{\mathrm{p}} L_{\mathrm{p}}\right) .\end{array}$ \\
\hline$S_{\mathrm{s} 11, \mathrm{~s} 12, \mathrm{~s} 21, \mathrm{~s} 22}$ & Scattering coefficients [22] & Varies \\
\hline$t_{\mathrm{a}, \mathrm{g}, \mathrm{p}}$ & $\begin{array}{l}\text { Single pass transmission } \\
\text { factor of the air gap, active } \\
\text { and passive cavity }\end{array}$ & $\begin{array}{c}t_{\mathrm{a}, \mathrm{g}, \mathrm{p}}= \\
\exp \left(-j \tilde{\beta}_{\mathrm{a}, \mathrm{g}, \mathrm{p}} L_{\mathrm{a}, \mathrm{g}, \mathrm{p}}\right)\end{array}$ \\
\hline$\tilde{\beta}_{\mathrm{a}, g, \mathrm{p}}$ & $\begin{array}{l}\text { Propagation constant of the } \\
\text { air gap, active and passive } \\
\text { cavity }\end{array}$ & $\begin{array}{c}\tilde{\beta}_{\mathrm{a}, \mathrm{g}, \mathrm{p}}= \\
\beta_{\mathrm{a}, \mathrm{g}, \mathrm{p}}-j \alpha_{\mathrm{a}, \mathrm{g}, \mathrm{p}} / 2\end{array}$ \\
\hline$L_{\mathrm{a}, \mathrm{g}}$ & $\begin{array}{l}\text { Active cavity and air gap } \\
\text { length }\end{array}$ & $1.5 \mathrm{~mm}$ and $13 \mu \mathrm{m}$ \\
\hline$L_{\mathrm{p}}$ & Passive cavity length & $\begin{array}{c}0.25 \mathrm{~mm}, 1.4 \mathrm{~mm} \text {, and } 3.4 \\
\mathrm{~mm}\end{array}$ \\
\hline$\beta_{\mathrm{a}, \mathrm{g}, \mathrm{p}}$ & $\begin{array}{l}\text { Real part of the propagation } \\
\text { constant }\end{array}$ & $\begin{array}{c}\beta_{\mathrm{a}, \mathrm{g}, \mathrm{p}}= \\
2 \pi n_{\mathrm{a}, \mathrm{g}, \mathrm{p}} \nu_{m} / \mathrm{c}\end{array}$ \\
\hline$\alpha_{\mathrm{a}, \mathrm{g}, \mathrm{p}}$ & $\begin{array}{l}\text { Net waveguide loss of the air } \\
\text { gap, active and passive cavity }\end{array}$ & $\begin{array}{c}9 \mathrm{~cm}^{-1}, \underset{\mathrm{cm}^{-1}}{0 \mathrm{~cm}^{-1}} \text {, and } 9 \\
\text {. }\end{array}$ \\
\hline$n_{\mathrm{a}, \mathrm{g}, \mathrm{p}}$ & $\begin{array}{l}\text { Effective refractive index of } \\
\text { the active and passive cavity }\end{array}$ & $3.61,1.0,3.61$ \\
\hline$\varphi_{\text {reff }}$ & Phase angle of $r_{\mathrm{eff}}$ & Varies \\
\hline$\nu_{m}$ & $\begin{array}{l}\text { Eigenmode frequency for } \\
\text { mode } m\end{array}$ & Varies \\
\hline$\alpha_{\mathrm{m}}$ & Effective mirror loss & $\begin{array}{l}\alpha_{\mathrm{m}}=\left(1 / L_{\mathrm{a}}\right) \\
\ln \left[1 /\left(\mathrm{r}_{1}\left|\mathrm{r}_{\mathrm{eff}}\right|\right)\right]\end{array}$ \\
\hline$\Gamma g_{\mathrm{th}}$ & Threshold modal gain & $\Gamma g_{\mathrm{th}}=\alpha_{\mathrm{a}}+\alpha_{\mathrm{m}}$ \\
\hline$\tau_{\mathrm{p} m}$ & Photon lifetime for mode $m$ & $\begin{array}{c}\tau_{\mathrm{p} m}= \\
1 /\left[v_{\mathrm{g} m}\left(\alpha_{\mathrm{a}}+\alpha_{\mathrm{m}}\right)\right]\end{array}$ \\
\hline$v_{\mathrm{g} m}$ & Group velocity of mode $m$ & Varies \\
\hline$\eta_{3}$ & Injection efficiency into ULL & $54.41 \%$ \\
\hline$\eta_{2}$ & Injection efficiency into LLL & $1.65 \%$ \\
\hline$\eta_{m}$ & $\begin{array}{l}\text { Power output coupling } \\
\text { coefficient for mode m }\end{array}$ & Varies \\
\hline$I_{\mathrm{a}}$ & Active cavity current & $0.75 \mathrm{~A}$ \\
\hline$I_{\mathrm{p}}$ & Passive cavity current & Varies \\
\hline$G_{\mathrm{m}}$ & Gain factor of mode $m$ & $\begin{array}{c}G_{\mathrm{m}}=G_{\mathrm{p}} /\left[1+\left(\left(\nu_{\mathrm{p}}-\right.\right.\right. \\
\left.\left.\left.\nu_{m}\right) /(\Delta \nu / 2)\right)^{2}\right]\end{array}$ \\
\hline$G_{\mathrm{p}}$ & $\begin{array}{l}\text { Peak gain factor of the } \\
\text { Lorentzian gain spectrum }\end{array}$ & $5.3 \times 10^{4} \mathrm{~s}^{-1}$ \\
\hline$\nu_{p}$ & Peak gain frequency & $2.795 \mathrm{THz}$ \\
\hline$\Delta \nu$ & FWHM of gain spectrum & $500 \mathrm{GHz}$ \\
\hline$M$ & $\begin{array}{l}\text { Number of periods in active } \\
\text { cavity }\end{array}$ & 90 \\
\hline$\beta_{\mathrm{sp}}$ & Spontaneous emission factor & $1.627 \times 10^{-4}$ \\
\hline$\tau_{3}$ & Total carrier lifetime in ULL & $5 \times 10^{-12} \mathrm{~s}$ \\
\hline$\tau_{32}$ & $\begin{array}{l}\text { Non-radiative relaxation time } \\
\text { from ULL to LLL }\end{array}$ & $1.76 \times 10^{-10} \mathrm{~s}$ \\
\hline$\tau_{2}$ & Total carrier lifetime in LLL & $2.1 \times 10^{-11} \mathrm{~s}$ \\
\hline$\tau_{\mathrm{sp}}$ & $\begin{array}{l}\text { Spontaneous emission } \\
\text { lifetime }\end{array}$ & $5 \times 10^{-10} \mathrm{~s}$ \\
\hline$R_{\mathrm{th}}$ & Thermal resistance & $9.92 \mathrm{~K} / \mathrm{W}$ \\
\hline$q$ & Electronic charge & $1.6 \times 10^{-19} \mathrm{C}$ \\
\hline$\hbar$ & Reduced Planck constant & $6.58 \times 10^{-16} \mathrm{eVs}$ \\
\hline
\end{tabular}

where $N_{3}(t)$ and $N_{2}(t)$ are the carrier populations in the upper and lower laser levels (ULL/LLL), respectively, and $S_{m}(t)$ represents the photon population in mode $m$. Equation (4) contains $N$ photon population equations for the longitudinal eigenmodes. The parameters $\left(\eta_{3}, \eta_{2}, G_{\mathrm{p}}, M, \beta_{\mathrm{sp}}, \tau_{3}, \tau_{32}, \tau_{2}\right.$,
TABLE I

DEVICE PARAMETERS USED IN NUMERICAL SimULATIONS (UNLESS STATED OTHERWISE)

and $\left.\tau_{\mathrm{sp}}\right)$ used in the equations are summarized in Table I The electron transport parameters are obtained from the full self-consistent energy-balance Schrödinger-Poisson scattering transport calculation for the given lasing cavity current $I_{\mathrm{a}}$ described in [24], [25]. The dependence of RRE parameters on the temperature and terminal voltage should be considered if $I_{\mathrm{a}}$ varies. However, $I_{\mathrm{a}}$ is fixed at $0.75 \mathrm{~mA}$ in this work. Therefore the parameters mentioned above are all constant as shown in Table I. Once solved, the emission output power can be obtained 
by $P_{m}(t)=\eta_{\mathrm{m}} h \nu_{m} S_{m}(t) / \tau_{\mathrm{p} m}$. We focus on the steady-state solutions of the multi-mode RRE in order to investigate mode selection and frequency tunability in CC THz QCL.

Equation (5) is the thermal model which describes the lattice temperature in the passive cavity $T_{\mathrm{p}}(t)$ as functions of the chip mass $m_{\mathrm{p}}$, the effective specific heat capacity $c_{\mathrm{p}}$ and the cold finger temperature $T_{0}$. Here we only show the thermal model for the passive cavity, but that for the active cavity should be included if $I_{\mathrm{a}}$ varies. By setting (5) to zero, the steady state solution is given by $\Delta T_{\mathrm{p}}=I_{\mathrm{p}} V_{\mathrm{p}} R_{\mathrm{th}}$, where $R_{\mathrm{th}}$ is the thermal resistance, and $I_{\mathrm{p}}$ and $V_{\mathrm{p}}$ are the current amplitude and voltage applied to the passive cavity, respectively [26]. Therefore, any tuning of the current at the passive cavity changes its lattice temperature, which in turn perturbs the refractive index of the passive cavity. This changes the reflectivity of the effective mirror, and can result in a mode hop of the dominant CC mode to a mode with the maximum net gain.

\section{RESUlts AND Discussions}

The dominant mode and the corresponding SMSR in a CC THz QCL are determined by both the device parameters in (1) and the RRE parameters of the RRE model (2)-(5). Using the scattering matrix method and the multi-mode RRE model, we have investigated the influence of the key parameters on mode selection in CC THz QCLs, including the net waveguide loss, effective refractive index of the passive cavity, and the heat sink temperature of the $\mathrm{CC}$ device. The Lorentzian gain spectrum with peak gain factor $G_{\mathrm{p}}$ at $2.795 \mathrm{THz}$ and full-width-halfmaximum (FWHM) of $500 \mathrm{GHz}$ [27] is applied in our simulation. Laser emission between $2.7 \mathrm{THz}$ and $2.85 \mathrm{THz}$ is our mode tuning range of interest. In reality, tuning one parameter will induce several parameters change simultaneously. In this section we change one parameter at a time, to gauge the individual effects of the various parameters over which we might hope to have some control. The usefulness of this method lies in the observation that the individual effects can be superimposed [21]. Via numerical simulation we can decouple these effects and understand how each parameter influences mode selection characteristics in CC THz QCL. The default device parameters, if not otherwise specified, are given in Table I. The effective refractive index of the waveguide active cavity is 3.61 at $2.7 \mathrm{THz}$ with the index dispersion $\mathrm{d} n / \mathrm{d} \nu=5.66 \times 10^{-14}(\mathrm{~Hz})^{-1}$, calculated using COMSOL Multiphysics. The net waveguide losses in the active and passive cavity are assumed to be invariant for the different eigenmodes, for simplicity.

\section{A. Comparison Between Single-and Coupled-Cavity QCLs}

We start our analysis with a comparison between the mode competition characteristics in a SC and CC THz QCL.The net transfer function and the threshold gain of a $1.5 \mathrm{~mm}$-long SC and a CC QCL with $L_{\mathrm{a}}=1.5 \mathrm{~mm}, L_{\mathrm{g}}=13 \mu \mathrm{m}$ and $L_{\mathrm{p}}=0.25 \mathrm{~mm}$ are shown in Fig. 2(a) and (b), respectively. The eigenmode frequencies for the SC QCL (black circles) start from $2.712 \mathrm{THz}$ with the mode spacing of $27.7 \mathrm{GHz}$, which is free spectral range (FSR) of the single cavity. The eigenmode frequencies of the CC QCL (red circles) are at 2.714, 2.74,

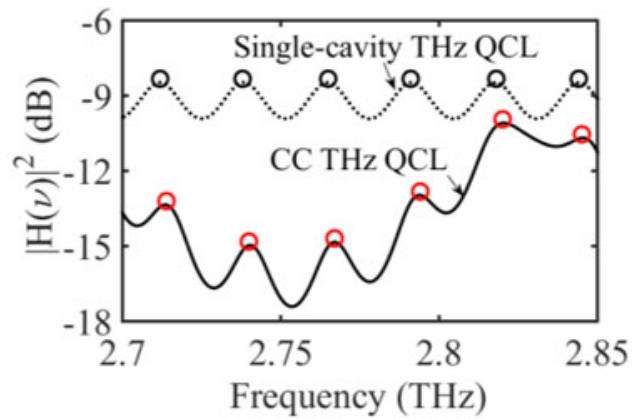

(a)

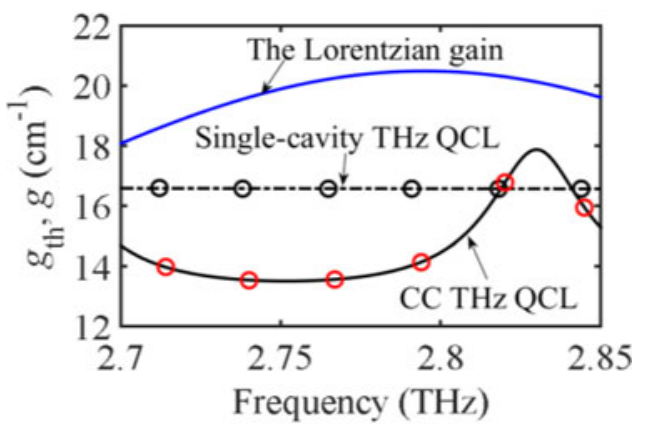

(b)

Fig. 2. Comparison of the (a) net transfer function and (b) the threshold gain between a 1.5-mm-long SC (in dotted lines) and a CC THz QCL ( $L_{\mathrm{a}}=1.5$ $\mathrm{mm}, L_{\mathrm{g}}=13 \mu \mathrm{m}$ and $L_{\mathrm{p}}=0.25 \mathrm{~mm}$ ) (in solid lines). The black and red circles indicate the eigenmodes for the SC and CC THz QCL, respectively. The Lorentzian gain is shown in solid blue in plot (b).

$2.767,2.794,2.82,2.845$, and $2.872 \mathrm{THz}$ with the mode spacing predominantly determined by the FSR of the lasing cavity. The eigenmodes for a THz QCL are labelled from mode 1 to 6 with increasing frequency in the following discussions. The threshold gain (the total loss) are identical for the eigenmodes in the SC QCL if neglecting the net waveguide loss dispersion, as shown in Fig. 2(b). The CC structure leads to a frequencydependent threshold gain, which exhibits a modulation with a period determined by the FSR of the passive cavity $(166.2 \mathrm{GHz}$ ). It is observed from Fig. 2(b) that the maxima of the net gain, $g_{\mathrm{n}}(\nu)=g(\nu)-g_{\mathrm{th}}(\nu)$ are at mode $4(2.791 \mathrm{THz})$ for the SC and at mode $3(2.757 \mathrm{THz})$ for the $\mathrm{CC}$ case.

In order to verify the dominant mode predicted from the linear cavity resonator analysis above, the emission spectrum with $I_{\mathrm{a}}=0.75 \mathrm{~A}$ for the SC and CC QCL are obtained by solving the multi-mode RRE and shown in Fig. 3(a) and (b), respectively. The dominant mode results are consistent with the prediction above. In addition, SMSR of $27.73 \mathrm{~dB}$ and $32.18 \mathrm{~dB}$ are obtained for the $\mathrm{SC}$ and $\mathrm{CC} \mathrm{THz}$ QCL, respectively, as shown in Fig. 3.

\section{B. Mode Competition Dependences on the Key Device Parameters in CC THz QCLs}

1) The Influence of Passive Cavity Net Waveguide Loss $\alpha_{\mathrm{p}}$ : Net waveguide loss in the passive cavity $\alpha_{\mathrm{p}}$ is defined as $<$ $\alpha_{i \mathrm{p}}>-<g_{\mathrm{p}}>$, where $<\alpha_{i \mathrm{p}}>$ and $<g_{\mathrm{p}}>$ are the average internal waveguide loss and modal gain in the passive cavity, 


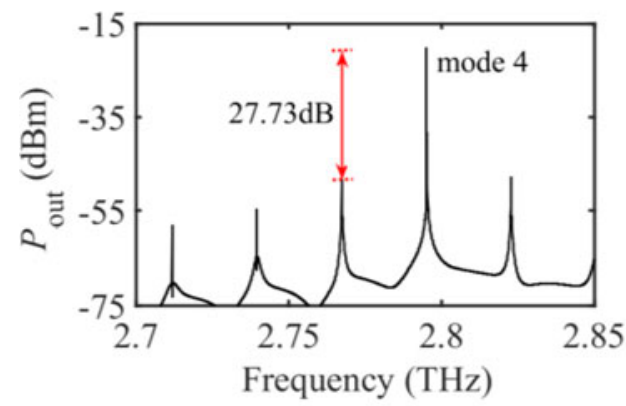

(a)

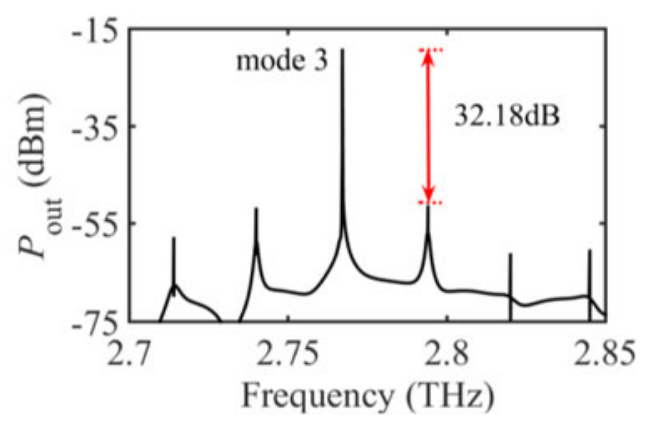

(b)

Fig. 3. The emission spectrum with $I_{\mathrm{a}}=0.75$ A for (a) the 1.5 -mm-long SC and (b) the CC THz QCL ( $L_{\mathrm{a}}=1.5 \mathrm{~mm}, L_{\mathrm{g}}=13 \mu \mathrm{m}$ and $\left.L_{\mathrm{p}}=0.25 \mathrm{~mm}\right)$.

respectively. Therefore, positive and negative $\alpha_{\mathrm{p}}$ indicate that modal gain is smaller and larger than the internal waveguide loss in the passive cavity, respectively. The passive cavity starts lasing when its modal gain overcomes the total loss of the passive cavity (the internal waveguide loss plus the mirror loss of the passive cavity $\alpha_{\mathrm{mp}}$ ), namely $\alpha_{\mathrm{p}}=-\alpha_{\mathrm{mp}}$. Our model is valid when the active cavity is lasing and passive cavity is not [21], so in the following discussions we limited $\alpha_{\mathrm{p}} \geq-\alpha_{\mathrm{mp}}$. $\alpha_{\mathrm{mp}}$ is 23,4 , and $1.7 \mathrm{~cm}^{-1}$ when $L_{\mathrm{p}}$ is $0.25 \mathrm{~mm}, 1.4 \mathrm{~mm}$ and 3.4 $\mathrm{mm}$, respectively. The net waveguide loss $\alpha_{\mathrm{p}}$ has a significant influence on the modulation depth of the frequency-dependent threshold gain $g_{\mathrm{th}}$ in a CC THz QCL. Varying modulation depth of $g_{\text {th }}$ induces the net gain relationship among the eigenmodes change, which in turn alters the dominant mode and its SMSR.

Fig. 4 summarizes the dependence of mode selection characteristics on $\alpha_{\mathrm{p}}$ for different passive cavity lengths $L_{\mathrm{p}}$. When $L_{\mathrm{p}}=0.25 \mathrm{~mm}$, the threshold gain spectra $g_{\mathrm{th}}$ with $\alpha_{\mathrm{p}}$ of -23 , -12 and $9 \mathrm{~cm}^{-1}$ are shown in Fig. 4(a1), where the eigenmodes are marked by circles. When $\alpha_{\mathrm{p}}$ is $-12 \mathrm{~cm}^{-1}, g_{\mathrm{th}}$ exhibits a sharp increase at the eigenmode frequency of $2.831 \mathrm{THz}$. The sharp peak decreases close to the baseline of $12.5 \mathrm{~cm}^{-1}$ when $\alpha_{\mathrm{p}}$ is $-23 \mathrm{~cm}^{-1}$ (reaching the lasing threshold of the passive cavity) and is very similar to the case of a single cavity. The peak value of $g_{\mathrm{th}}$ with $\alpha_{\mathrm{p}}$ of $9 \mathrm{~cm}^{-1}$ is intermediate. In order to show how the $g_{\mathrm{th}}$ profile varies with $\alpha_{\mathrm{p}}$, the peak value of $g_{\mathrm{th}}$ as a function of $\alpha_{\mathrm{p}}$ at $2.831 \mathrm{THz}$ is shown in Fig. 4(a2). There is a maximum when $\alpha_{\mathrm{p}}$ is $-12 \mathrm{~cm}^{-1}$, determined by (1). Increasing the peak value of $g_{\text {th }}$ results in maximum net gain transfers from mode 5 to mode 3, which is verified by the emission spectra shown in Fig. 4(a3). It is noted that there is no emission at mode 5 of $2.831 \mathrm{THz}$ when $\alpha_{\mathrm{p}}$ is $-12 \mathrm{~cm}^{-1}$ [red dotted curve in Fig. 4(a3)] due to its gain $\left(20.1 \mathrm{~cm}^{-1}\right)$ being below threshold. Furthermore, it can be predicted that at $2.831 \mathrm{THz}$, lasing is not possible when $\alpha_{\mathrm{p}}$ is between -17 and $1 \mathrm{~cm}^{-1}$, as indicated by the dotted line in Fig. 4(a2). We can conduct the same analysis for other modes to determine the potential tuning range of $\alpha_{\mathrm{p}}$ that supports lasing.

When $L_{\mathrm{p}}$ and $L_{\mathrm{a}}$ are comparable as in the case of Fig. 4(b), the eigenmodes are spaced by about the same amount with the modulation period of $g_{\mathrm{th}}$. When $\alpha_{\mathrm{p}}$ is -4 and $9 \mathrm{~cm}^{-1}$, the $g_{\mathrm{th}}$ differences between eigenmodes are small so that the dominant mode is mainly determined by the gain spectrum peak at 2.795
THz. In addition, the SMSR in both cases are smaller compared with the results of $L_{\mathrm{p}}<L_{\mathrm{a}}$ shown in Fig. 4(a3). The gain at each eigenmode is below its threshold when $\alpha_{\mathrm{p}}$ is $-2 \mathrm{~cm}^{-1}$ so that the emission power are all less than $-55 \mathrm{dBm}$ in Fig. 4(b3) (red dotted curve). Also, we found that mode 5 at $2.814 \mathrm{THz}$ is not able to lase when $\alpha_{\mathrm{p}}$ is between -3 to $-0.2 \mathrm{~cm}^{-1}$.

In the third case with $L_{\mathrm{p}}>L_{\mathrm{a}}$ as in Fig. 4(c), $\alpha_{\mathrm{p}}$ with the local maxima of the peak value of $g_{\mathrm{th}}$ increased to $-0.9 \mathrm{~cm}^{-1}$ (c2) where $\mathrm{THz}$ emission at mode 3 is observed while other modes are suppressed ( $\mathrm{c} 1$ and $\mathrm{c} 3$ ). However, with the decreased peak amplitude of $g_{\mathrm{th}}$ when $\alpha_{\mathrm{p}}$ is -1.7 and $9 \mathrm{~cm}^{-1}$ (blue dash and dotted and red dotted lines in $\mathrm{c} 1$ ), the dominant mode changes to near gain spectrum peak at $2.793 \mathrm{THz}$ and $2.82 \mathrm{THz}$, respectively (c3). It is obtained from c2 that mode 11 at 2.828 THz is not able to lase when $\alpha_{\mathrm{p}}$ is between -1.2 to $-0.1 \mathrm{~cm}^{-1}$.

Comprehensive simulation reveals that the main eigenmode frequencies are $2.714,2.74,2.767,2.794,2.821,2.845$ and $2.872 \mathrm{THz}$ for $L_{\mathrm{p}}$ are values of $0.25 \mathrm{~mm}, 1.4 \mathrm{~mm}$ and 3.4 $\mathrm{mm}$. However, they vary when $\alpha_{\mathrm{p}}$ is in the rising regions in Fig. 4(a2), (b2) and (c2). When $L_{\mathrm{p}}=0.25 \mathrm{~mm}$, the two eigenmodes of $2.821 \mathrm{THz}$ and $2.845 \mathrm{THz}$ merge into one mode at $2.83 \mathrm{THz}$ when $\alpha_{\mathrm{p}}$ is between $-23 \sim-10 \mathrm{~cm}^{-1}$. When $L_{\mathrm{p}}=1.4 \mathrm{~mm}$ with $\alpha_{\mathrm{p}}$ of $-4 \sim 10 \mathrm{~cm}^{-1}$, all eigen modes decrease about $10 \mathrm{GHz}$. When $L_{\mathrm{p}}=3.4 \mathrm{~mm}$ with $\alpha_{\mathrm{p}}$ of $-1.7 \sim 7 \mathrm{~cm}^{-1}$, the eigenmode spacing decreases to around $12 \mathrm{GHz}$, which is close to the FSR of the passive cavity. By using this model, one can obtain the net waveguide loss values that allow each eigenmode lases. In addition, by controlling the modulation depth of threshold gain spectrum through net waveguide loss, one can design the CC QCL device to lase at a desired eigenmode with good SMSR above $30 \mathrm{~dB}$, also achieve mode tuning by control of the passive current amplitude to tune the net waveguide loss of the passive cavity. When tuning $\alpha_{\mathrm{p}}$, it is observed that $L_{\mathrm{a}} \sim L_{\mathrm{p}}$ is more stable with less tunability and $L_{\mathrm{a}}<L_{\mathrm{p}}$ has better mode tunability but with reduced SMSR. The mode tunability of $L_{\mathrm{a}}>L_{\mathrm{p}}$ is intermediate, with good SMSR above 30 dB.

2) The Influence of Passive Cavity Optical Length $n_{\mathrm{p}} L_{\mathrm{p}}$ : Optical length of the passive cavity $n_{\mathrm{p}} L_{\mathrm{p}}$ determines phase of the $g_{\text {th }}$ spectrum through the effective mirror reflection coefficient $r_{\text {eff }}$. Therefore, perturbation of the effective refractive index of the passive cavity induces a phase shift of the $g_{\text {th }}$ spec- 

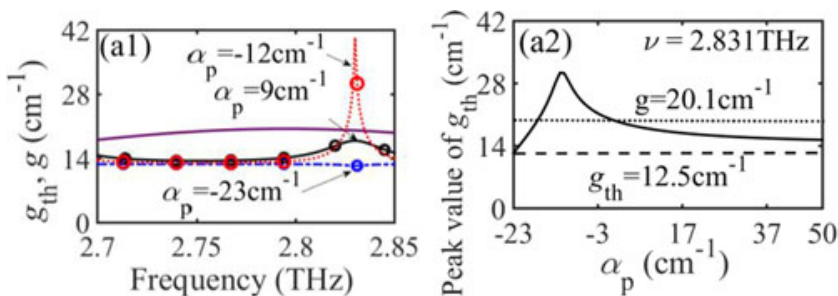

(a)
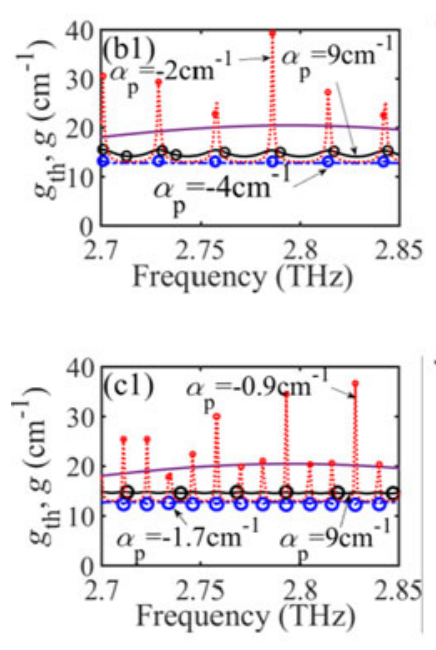

(b)

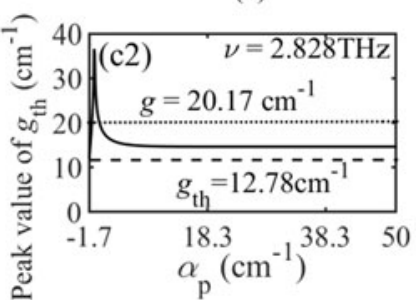

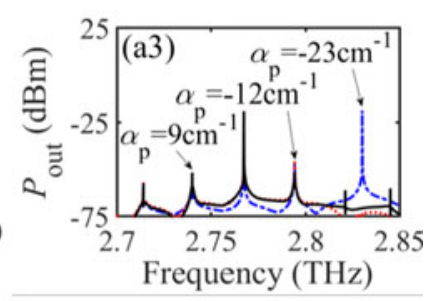

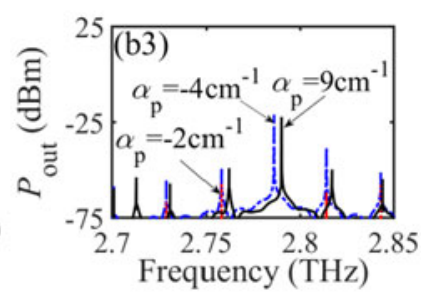

(c)

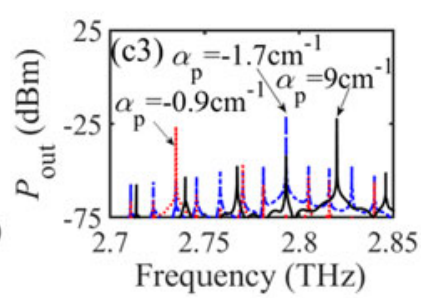

Fig. 4. The dependence of mode selection on the passive cavity net waveguide loss $\alpha_{\mathrm{p}}$ for the long, medium and short passive cavities: (a) $L_{p}=0.25$ mm (b) $L_{p}=1.4 \mathrm{~mm}$ and (c) $L_{p}=3.4 \mathrm{~mm}$, where (a1), (b1) and (c1) show the threshold gains at various $\alpha_{\mathrm{p}}$ with the Lorentzian gain indicated by purple solid lines, the eigenmodes are marked by circles; (a2), (b2) and (c2) plot the peak value of $\mathrm{g}_{\mathrm{th}}$ as a function of $\alpha_{\mathrm{p}}$, where the dashed lines indicate the active cavity g th when the total loss of passive cavity is exactly overcome by the modal gain of the passive cavity, and the dotted line indicate the active cavity gain at the eigenmode frequency showed in the plot; the emission spectra at various $\alpha_{\mathrm{p}}$ are shown in (a3), (b3) and (c3), respectively.
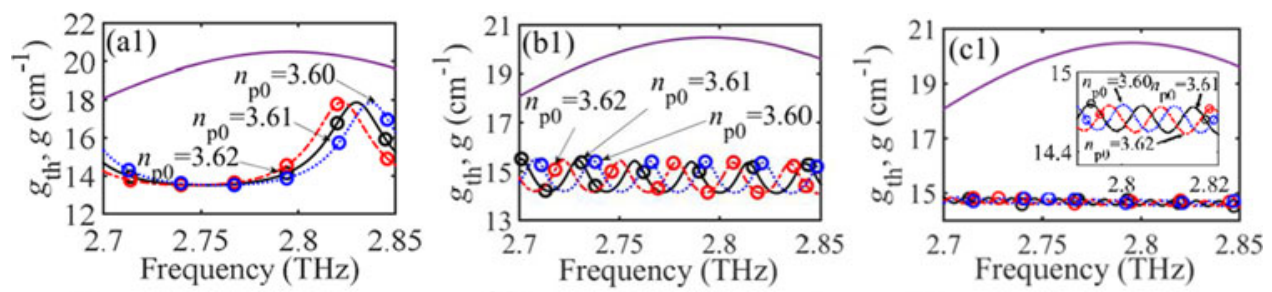

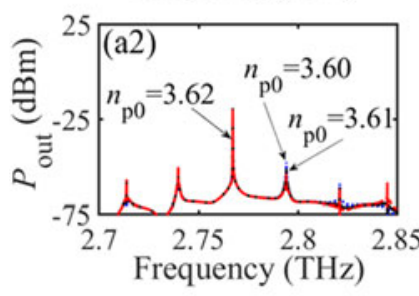

(a)

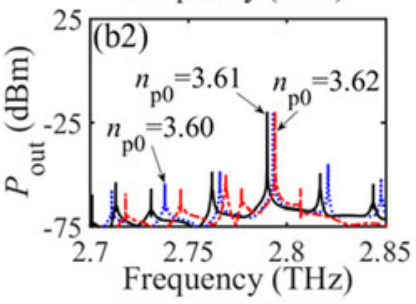

(b)

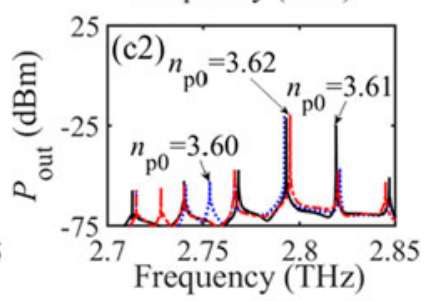

(c)

Fig. 5. The dependence of mode selection characteristics on the passive cavity effective refractive index (represented by the effective refractive index at 2.7 THz $n_{\mathrm{p} 0}$ ) for the long, medium and short passive cavities: (a) $L_{\mathrm{p}}=0.25 \mathrm{~mm}$ (b) $L_{\mathrm{p}}=1.4 \mathrm{~mm}$ and (c) $L_{\mathrm{p}}=3.4 \mathrm{~mm}$, where the Lorentzian gain spectra are indicated by purple solid lines, the threshold gains with $n_{\mathrm{p} 0}$ of $3.60,3.61$ and 3.62 are given in red dash and dotted, black solid and blue dotted lines, respectively in (a1), (b1) and (c1) with the eigenmodes marked by circles; the emission spectrum with $n_{\mathrm{p} 0}$ of $3.60,3.61$ and 3.62 are shown in red dash and dotted, black solid and blue dotted lines, respectively in (a2), (b2) and (c2), respectively.

trum. Depending on the phase angle of $r_{\text {eff }}$, red shift coefficient of $g_{\mathrm{th}}$ spectrum varies with $L_{\mathrm{p}}$. Tuning adjustments of 0.01 in the effective refractive index of the passive cavity about the reference value of $2.7 \mathrm{THz}, n_{\mathrm{p} 0}$, were found to cause red shifts of $7 \mathrm{GHz}$ in $g_{\mathrm{th}}$ for $L_{\mathrm{p}}=0.25 \mathrm{~mm}$ and $1.4 \mathrm{~mm}$ [Shown in Fig. 5(a1) and (b1)]. While the red shift coefficient when $L_{\mathrm{p}}=3.4$ $\mathrm{mm}$ is about $4 \mathrm{GHz} / 0.01$, as a function of $n_{\mathrm{p} 0}$, as shown in Fig. 5(c1). The eigenmode frequencies are invariant when $n_{\mathrm{p} 0}$ varies by \pm 0.01 and $L_{\mathrm{p}}=0.25 \mathrm{~mm}$, but have a slight shift when $L_{\mathrm{p}}=1.4 \mathrm{~mm}$ and $3.4 \mathrm{~mm}$, as indicated in Fig. 5(b1) and 


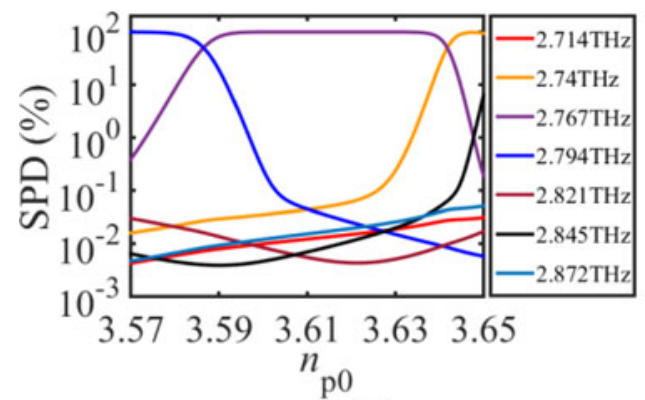

(a)

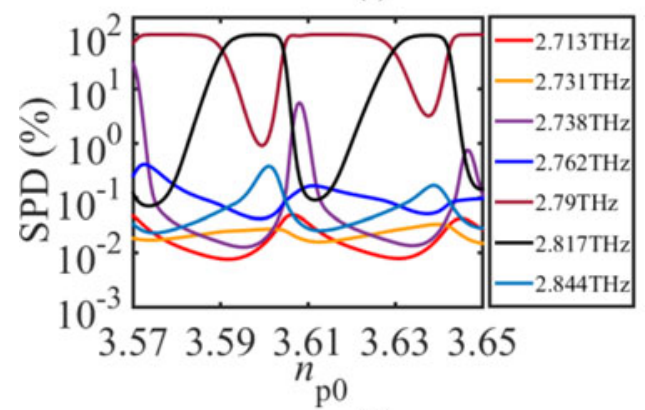

(b)

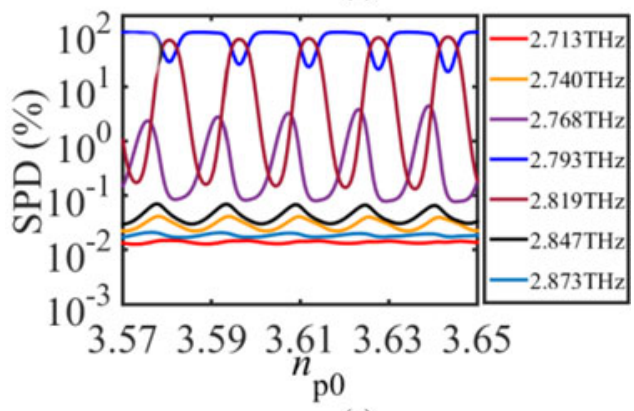

(c)

Fig. 6. The steady state SPD as a function of $n_{\mathrm{p} 0}$ for the long, medium and short passive cavities: (a) $L_{\mathrm{p}}=0.25 \mathrm{~mm}$; (b) $L_{\mathrm{p}}=1.4 \mathrm{~mm}$ and (c) $L_{\mathrm{p}}=3.4 \mathrm{~mm}$.

(c1). If we neglect small changes in the eigenmode frequencies, it is observed that there is no mode hopping for all these three passive cavity lengths, as shown in Fig. 5(a2), (b2) and (c2). This could be explained by the net gain at these frequencies having no significant change as a function of $n_{\mathrm{p} 0}$ as shown in (a1), (a2) and (a3), respectively.

Change in $n_{\mathrm{p} 0}$ reflects the influence of varying driving current applied to the passive cavity due to the injection heating effect, commonly used mechanism for frequency tuning in coupledcavity semiconductor lasers. The passive cavity current induced perturbation of the refractive index results in a change of the dominant mode and SMSR. We vary $n_{\mathrm{p} 0}$ over a wide range of \pm 0.04 to fully explore mode tunability via control of the passive cavity current. The result is the number of mode switching amongst different mode frequencies attainable for a given tuning range. On the other hand, the passive cavity length may be adjusted to provide the desired mode tunability. The steady state SPDs as a function of $n_{\mathrm{p} 0}$ (varying by \pm 0.04 ) for $L_{\mathrm{p}}=0.25$ $\mathrm{mm}, 1.4 \mathrm{~mm}$ and $3.4 \mathrm{~mm}$ are calculated and shown in Fig. 6(a), (b) and (c) respectively. Mode hopping amongst modes 4, 3 and 2 at 2.794, 2.767, 2.74 THz, respectively, with excellent SMR is observed with $n_{\mathrm{p} 0}$ varying from 3.57 to 3.65 in Fig. 6(a) when $L_{\mathrm{a}}>L_{\mathrm{p}}$. With the same variation of $n_{\mathrm{p} 0}$, periodical mode hopping between only two modes is observed for the cases $L_{\mathrm{a}}$ $\sim L_{\mathrm{p}}$ and $L_{\mathrm{a}}<L_{\mathrm{p}}$. As shown in Fig. 6(b), when $L_{\mathrm{p}}=1.4$ $\mathrm{mm}$, mode 5 at $2.79 \mathrm{THz}$ and mode 6 at $2.817 \mathrm{THz}$ alternately and gradually swap their power when $n_{\mathrm{p} 0}$ varies every 0.038 , whereas in Fig. 6(c) with $L_{\mathrm{p}}=3.4 \mathrm{~mm}$, mode $42.793 \mathrm{THz}$ and mode 5 at $2.819 \mathrm{THz}$ transfer their dominant roles more frequently variations of just 0.016 in $n_{\mathrm{p} 0}$. However, good SMSR is not obtained when mode 5 is dominant in this case. Again, the mode tuning results obtained from solving the nonlinear RREs could be well explained by the net gain relationship amongst the eigenmodes obtained from linear resonator analysis. As shown in Fig. 5(a1), the net gain differences between the eigenmodes when $L_{\mathrm{p}}<L_{\mathrm{a}}$ are much greater than those when $L_{\mathrm{p}} \sim L_{\mathrm{a}}$ and $L_{\mathrm{p}}>L_{\mathrm{a}}$ due to the longer modulation period of $g_{\mathrm{th}}$. We predict that mode tuning amongst more eigenmodes would occur when $L_{\mathrm{a}}>L_{\mathrm{p}}$ with greater variation in $n_{\mathrm{p} 0}$. However, due to the typical peak gain generally being much higher than $g_{\text {th }}$ when $L_{\mathrm{a}} \sim L_{\mathrm{p}}$ and $L_{\mathrm{a}}<L_{\mathrm{p}}$ in CC THz QCLs, as shown in Fig. 5(b1) and (c1), the maximum net gain is always around the peak gain at $2.795 \mathrm{THz}$. For this reason the dominant modes for $L_{\mathrm{p}}=1.4$ $\mathrm{mm}$ and $3.4 \mathrm{~mm}$ are always between $2.79 \mathrm{THz}$ and $2.82 \mathrm{THz}$ in Fig. 6(b) and (c).

Furthermore, the eigenmode distribution in the $g_{\text {th }}$ spectrum determines the mode tunability as well. Suppose for instance that, one of the two dominant modes lies at rising edge and the other at falling edge of $g_{\mathrm{th}}$ so that a red phase shift results in net gain of the mode at rising edge decreasing, while that of the mode at falling edge increases simultaneously. This scenario is illustrated by the two eigenmodes at 2.793 and $2.819 \mathrm{THz}$ when $n_{\mathrm{p} 0}=3.60$, as shown in the inset of Fig. 5(c1) with blue circles. This process gives rise to mode swapping only between these two modes when $n_{\mathrm{p} 0}$ varies from 3.57 to 3.65 where $g_{\mathrm{th}}$ has a red shift of two full cycles. Therefore, no matter how wide the tuning range of $n_{\mathrm{p} 0}$, mode switching is only possible between two modes close to the gain spectrum peak in Fig. 6(b) and (c). In addition, if both of the eigenmodes lie at rising or falling edge of $g_{\text {th }}$ profile, tuning $n_{\mathrm{p} 0}$ might not change the dominant mode since their net gain relationship would not change. Based on the mode selection characteristic analysis in CC THz QCLs above, we found that in order to enhance mode tunability to obtain mode switching among more modes when $L_{\mathrm{a}} \sim L_{\mathrm{p}}$ and $L_{\mathrm{a}}<L_{\mathrm{p}}$, we can either decrease the gain spectrum peak or increase the threshold gain so as to increase the net gain differences among the eigenmodes. Alternatively, we can increase the modulation depth of the threshold gain to enhance the net gain differences by controlling the net waveguide loss as discussed in Section IV-B1. Furthermore, with a certain gain spectrum, the desired eigenmodes should distributed at different edges of the $g_{\text {th }}$ profile so that their net gain changes with $n_{\mathrm{p} 0}$ asynchronously. Last but not least, given a limited passive current tuning range, adjusting the effective refractive index could be replaced by altering cavity length at the design stage as a much easier way to enhance the mode tuning capability. For instance, for a CC THz QCL with $n_{\mathrm{p} 0}=3.61$ and $L_{\mathrm{p}}=0.25$ $\mathrm{mm}$, if the passive cavity current applied to the passive cavity is varied from 0-2 A, which induced lattice temperature in the passive cavity varies from $0-195 \mathrm{~K}$. This in turn results in $n_{\mathrm{p} 0}$ 

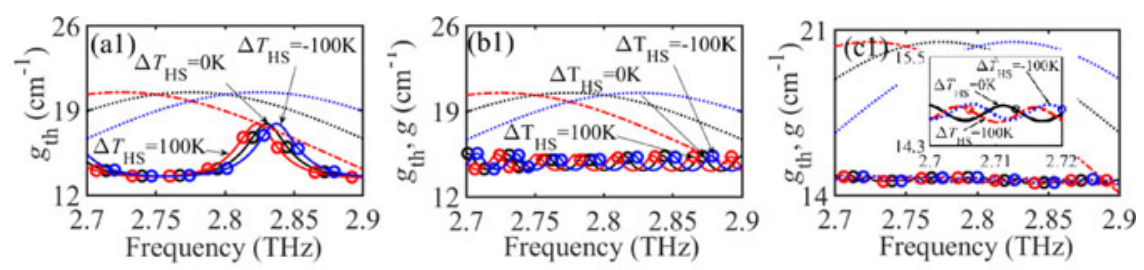

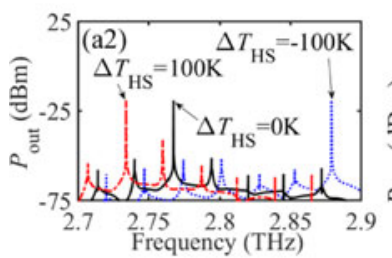

(a)

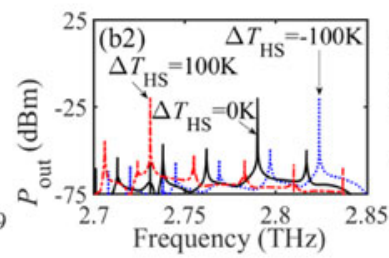

(b)

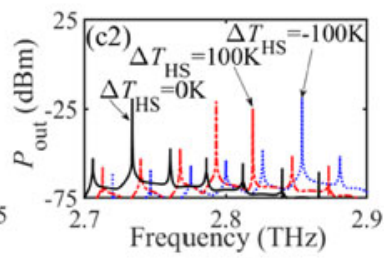

(c)

Fig. 7. Threshold gains and the emission spectra for heat sink temperature variation of $\pm 100 \mathrm{~K}$ in CC THz QCLs for the long, medium and short passive cavities: (a) $L_{\mathrm{p}}=0.25 \mathrm{~mm}$; (b) $L_{\mathrm{p}}=1.4 \mathrm{~mm}$; (c) $L_{\mathrm{p}}=3.4 \mathrm{~mm}$.
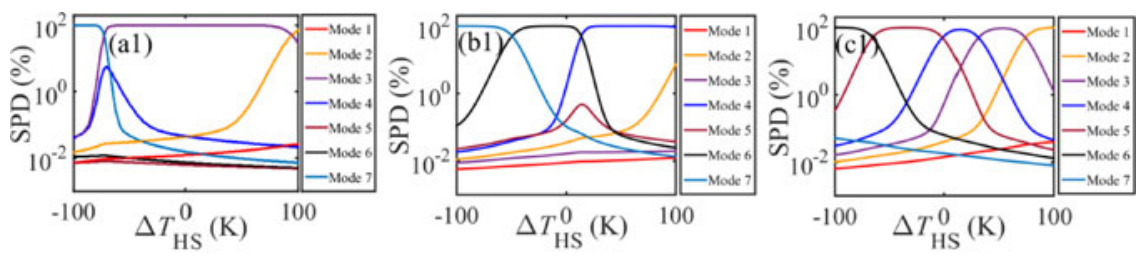

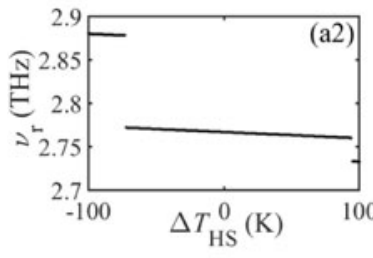

(a)

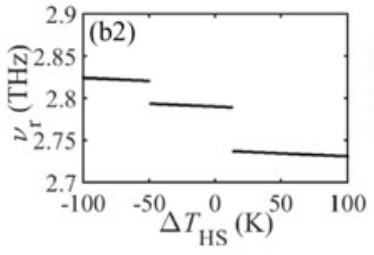

(b)

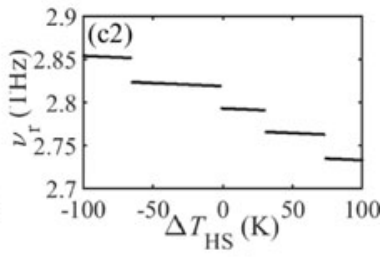

(c)

Fig. 8. The steady state SPD and the dominant mode frequency $\nu_{\mathrm{r}}$ as a function of the heat sink temperature variation for the long, medium and short passive cavities: (a) $L_{\mathrm{p}}=0.25 \mathrm{~mm}$; (b) $L_{\mathrm{p}}=1.4 \mathrm{~mm}$; (c) $L_{\mathrm{p}}=3.4 \mathrm{~mm}$.

changes from 3.61 to 3.63 where no mode tuning occurs as shown in Fig. 6(a). However, if we increase $L_{\mathrm{p}}$ to around 0.252 $\mathrm{mm}$ (corresponding to tuning $n_{\mathrm{p} 0}$ to 3.642 in Fig. 6(a), where mode hopping occurs), tuning between modes 3 and 2 can be obtained with a lesser tuning range for $n_{\mathrm{p} 0}$. Therefore, we can choose an appropriate passive cavity length to suit a limited current tuning range in order to obtain mode tuning.

3) The Dependence on the Heat Sink Temperature $T_{\mathrm{HS}}$ : When the heat sink temperature varies, on one hand, the refractive index of both the active cavity and the passive cavity, $n_{\mathrm{a} 0}$ and $n_{\mathrm{p} 0}$, varies with temperature coefficient $d \mathrm{n} / \mathrm{d} T_{\mathrm{HS}}=$ $9.5 \times 10^{-5}$ [18]. Simultaneously, the gain spectrum peak has a red shift with the coefficient of around $500 \mathrm{MHz} / \mathrm{K}$ on average [28]. Consequently, the eigenmode frequencies red shift with a temperature coefficient of $60-80 \mathrm{MHz} / \mathrm{K}$ due to variance in $n_{\mathrm{a} 0}$, which is consistent with the $63-90 \mathrm{MHz} / \mathrm{K}$ reported in [18], whilst the threshold gain has a red shift coefficient of 70 $\mathrm{MHz} / \mathrm{K}$ on average as predicted from our simulation. Therefore the eigenmode frequencies, threshold gain and gain peak shift at different rates with temperature, which leads to mode tuning.

In order to show the mode tuning process induced by control of the heat sink temperature, the threshold gain and emis- sion spectra with a heat sink temperature variation $\Delta T_{\mathrm{HS}}$ of $\pm 100 \mathrm{~K}$, and with $L_{\mathrm{p}}=0.25 \mathrm{~mm}, 1.4 \mathrm{~mm}$, and $3.4 \mathrm{~mm}$ are depicted in Fig. 7(a), (b) and (c), respectively. The net gain maximum has red shifts from mode 7 at $2.879 \mathrm{THz}$ to mode 3 at $2.767 \mathrm{THz}$, then to mode 2 at $2.733 \mathrm{THz}$ when $\Delta T_{\mathrm{HS}}$ is $-100 \mathrm{~K}$, $0 \mathrm{~K}$ and $100 \mathrm{~K}$, respectively, as shown in Fig. 7(a1). The results are consistent with the emission spectra shown in Fig. 7(a2). With $\Delta T_{\mathrm{HS}}$ values of $-100 \mathrm{~K}, 0 \mathrm{~K}$ and $100 \mathrm{~K}$ corresponding to $n_{\mathrm{p} 0}$ of $3.60,3.61$ and 3.62 , respectively, no mode hopping is observed from the emission spectrum shown in Fig. 5(a2). Similar comparisons with each pair of passive cavity lengths give the same result. Mode hopping among more modes could be obtained by control of the heat sink temperature compared with only tuning the passive cavity current. The red shift of both gain spectrum peak and eigenmode frequencies play an important role in mode switching. And the eigenmode shifting leads to continuous frequency tuning of the dominant mode as well. The updated net gains among the altered eigenmodes determine the next dominant mode and the corresponding SMSR.

Even though $\mathrm{THz}$ QCLs with the operation temperatures varying from $8 \mathrm{~K}$ to $200 \mathrm{~K}$ is now available [8], the output powers at higher temperature would be very low. However, by 


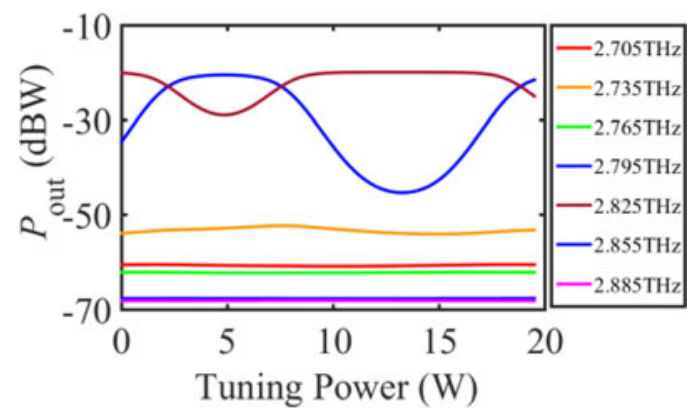

(a)

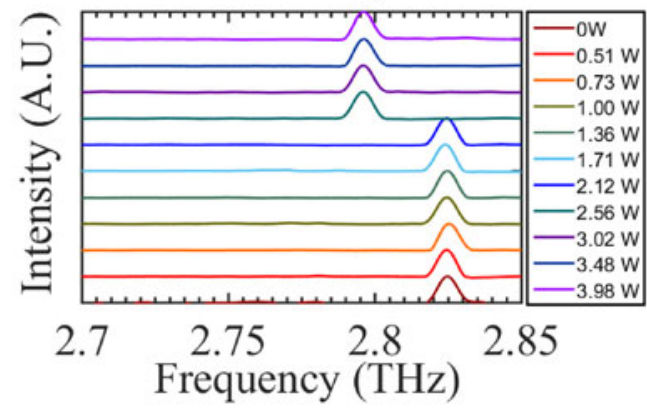

(b)

Fig. 9. Comparison of the mode tuning by passive cavity current between (a) the numerical simulation result and (b) the experimental data.

varying the heat sink temperature $\Delta T_{\mathrm{HS}}$ at a wide tuning range from $-100 \mathrm{~K}$ to $100 \mathrm{~K}$, allows us to fully explore the effect on frequency tuning ability (amongst how many different modes) and thus to make a choice of heat sink temperature or peak gain frequency that provide a suitable combination of mode switches and continuous frequency tuning. For real $\mathrm{CC} \mathrm{THz} \mathrm{devices,} \mathrm{the}$ active and passive cavity temperatures can also be manipulated by careful selection of excitation pulse duration and magnitude, as well as the thermal resistance of chip to submount interface, which has a marked impact on overall temperature change.

Fig. 8(a), (b) and (c) depict the steady state SPD as a function of $\Delta T_{\mathrm{HS}}$ for $L_{\mathrm{p}}=0.25 \mathrm{~mm}, 1.4 \mathrm{~mm}$ and $3.4 \mathrm{~mm}$, respectively. The change of the dominant mode frequencies $\nu_{\mathrm{r}}$ as a function of $\Delta T_{\mathrm{HS}}$ are plotted as well, to illustrate the continuous frequency tuning of the dominant modes. We find that more mode hopping occurs when varying $\Delta T_{\mathrm{HS}}$ from $-100 \mathrm{~K}$ to $100 \mathrm{~K}$ in shown in Fig. 8(a), (b) and (c), than when $n_{\mathrm{p} 0}$ varies from 3.60 to 3.62, as shown in Fig. 6(a), (b) and (c), respectively. In addition, during each dominant mode period the dominant mode frequencies continuously red shift with increasing heat sink temperature. Mode hopping amongst 3 modes 7, 3 and 2, and a continuous frequency tuning of $11 \mathrm{GHz}$ in mode 3 is achieved in Fig. 8(a2). Mode hopping amongst 3 modes with continuous frequency tuning of $4 \mathrm{GHz}, 4 \mathrm{GHz}$ and $6 \mathrm{GHz}$ in each mode are realized when $L_{\mathrm{p}}=1.4 \mathrm{~mm}$, as shown in Fig. 8(b1) and (b2). When $L_{\mathrm{p}}=3.4 \mathrm{~mm}$, successive mode hopping from 6 to 2 with continuous frequency tuning from $2-5 \mathrm{GHz}$ are observed in Fig. 8(c1) and (c2). Therefore, in these cases with the same $\Delta T_{\mathrm{HS}}$, mode tunability is improved with $L_{\mathrm{a}}<L_{\mathrm{p}}$ in this case compared with $L_{\mathrm{a}} \sim L_{\mathrm{p}}$ but with reduced SMSR.

From the analysis above, we demonstrate the sensitivity of the dominant mode, SMSR, as well as the emission frequency to the key device parameters in a CC THz QCL for different ratios of active cavity and passive cavity. Due to the longer active cavity length, the mirror loss is greatly reduced in the $\mathrm{CC} \mathrm{THz}$ QCLs compared with that in diode lasers. This results in a gain spectrum high above the threshold gain spectrum while they are comparable in diode lasers as demonstrated in [20]. Therefore, multiple modes have net gain above zero in CC THz QCLs, which makes the mode tuning characteristics more complicated. The mode competition and selection mechanism depend on the ratio of the lasing and passive cavity length and the eigenmode locations in the threshold gain spectrum as well. Frequency tuning in a $\mathrm{CC} \mathrm{THz} \mathrm{QCL} \mathrm{is} \mathrm{normally} \mathrm{realized} \mathrm{by} \mathrm{control} \mathrm{the}$ passive cavity current $I_{\mathrm{p}}$ for discrete mode hopping or the heat sink temperature $T_{\mathrm{HS}}$ for both mode hopping and continuous frequency tuning. It is observed that $L_{\mathrm{a}}>L_{\mathrm{p}}$ is more stable without mode hopping than $L_{\mathrm{a}} \sim L_{\mathrm{p}}$ and $L_{\mathrm{a}}<L_{\mathrm{p}}$ when tuning $I_{\mathrm{p}}$ or $T_{\mathrm{HS}}$, which is consistent with the case in CC diode lasers [20]. However, when tuning $I_{\mathrm{p}}$ over a wider range, depending on $L_{\mathrm{p}}, L_{\mathrm{a}}>L_{\mathrm{p}}$ exhibits mode tuning amongst more modes compared to the cases $L_{\mathrm{a}} \sim L_{\mathrm{p}}$ and $L_{\mathrm{a}}<L_{\mathrm{p}}$ when the eigenmodes near the gain peak are located at similar edges of the $g_{\text {th }}$ profile. Mode tuning among more modes could be realized when $L_{\mathrm{a}} \sim L_{\mathrm{p}}$ and $L_{\mathrm{a}}<L_{\mathrm{p}}$ if eigenmodes located at different edges or locations of $g_{\mathrm{th}}$ spectrum so that their net gain changes with $I_{\mathrm{p}}$ asynchronously. When tuning the heat sink temperature, continuous frequency tuning is observed for three different ratios of $L_{\mathrm{a}}$ and $L_{\mathrm{p}}$. However, a wider continuous frequency tuning range with a less discrete mode tuning could be obtained for the same variation of $T_{\mathrm{HS}}$ when $L_{\mathrm{a}}>L_{\mathrm{p}}$ compared with $L_{\mathrm{a}} \sim L_{\mathrm{p}}$ and $L_{\mathrm{a}}<L_{\mathrm{p}}$. Furthermore, frequency tunability could be enhanced with a proper combination of drive currents in both cavities and the heat sink temperature. There are a variety of sensing, spectroscopy, and imaging applications that require tunable $\mathrm{THz}$ laser sources [29]-[31]. By using the CC THz QCLs, we can control the convenient parameters (such as the cavity lengths, the injection currents, and the heat sink temperature) to achieve desired frequency tuning range. For example, discrete (continuous) tuning of $100 \mathrm{GHz}(10 \mathrm{GHz})$ has recently been achieved experimentally from CC QCLs through appropriate cavity length optimization [32].

\section{COMPARISON BETWEen Simulation REsult AND EXPERIMENTAL RESULT: MODEL VERIFICATION}

The theoretical model described above is verified by experimental data for mode tuning by controlling the tuning power applied on the passive cavity current (the product of $I_{\mathrm{p}}$ and terminal voltage $V_{\mathrm{p}}$ ). The comparison is carried for the fabricated CC QCL with $L_{\mathrm{a}}=1.5 \mathrm{~mm}, L_{\mathrm{g}}=13 \mu \mathrm{m}$ and $L_{\mathrm{p}}=$ $3.4 \mathrm{~mm}$ described in [18]. As shown in Fig. 9(a), mode switching between Mode 5 at $2.825 \mathrm{THz}$ and Mode 4 at $2.795 \mathrm{THz}$ is anticipated from the numerical simulation when the tuning power in the passive cavity varies from 0 to $3.8 \mathrm{~W}$, which agrees very well with the experimental data shown in Fig. 9(b), [18]. By using multi-mode RRE modelling, which involves the influence of the gain spectrum, more accurate results can be obtained than the simplified numerical approach described in [18], which 
is based on the scattering matrix method only. In addition, the simulation results predict that mode tuning is only available between the two modes even with wider tuning power above 3.8 W. By developing a scattering matrix method in conjunction with multi-mode RREs, complete modelling of multi-mode competition in CC QCLs is predicted and analysed.

\section{CONCLUSION}

Multi-mode reduced rate equations for THz QCLs were developed and the complete model for a coupled cavity $\mathrm{THz}$ QCL is established in this work. The accuracy of the proposed modelling approach has been validated with experimental data. Mode selection and frequency tuning capability of $\mathrm{CC} \mathrm{THz}$ QCLs are investigated for different ratios of the lasing and passive cavity lengths. The net waveguide loss and the refractive index of the passive cavity determined the modulation depth and the phase of the threshold gain, respectively. Increase in heat sink temperature leads to red shifting of eigenmode frequencies, the threshold gain spectrum and the gain spectrum at different rates. The dominant mode and the SMSR are determined by the updated net gain relationship among the modes. The longer cavity lengths of THz QCLs result in a gain spectrum peak high above the threshold gain, which makes mode competition more complicated than in diode lasers. For discrete mode tuning capability, $L_{\mathrm{a}}>L_{\mathrm{p}}$ is more stable than $L_{\mathrm{a}} \sim L_{\mathrm{p}}$ and $L_{\mathrm{a}}<L_{\mathrm{p}}$, where the eigenmode distributions in the threshold gain spectrum influences mode competition as well. $L_{\mathrm{a}}<L_{\mathrm{p}}$ is more mode tunable than $L_{\mathrm{a}} \sim L_{\mathrm{p}}$ but with reduced SMSR. For continuous frequency tunability, wider continuous frequency tuning range could be obtained when $L_{\mathrm{a}}>L_{\mathrm{p}}$ compared with $L_{\mathrm{a}} \sim L_{\mathrm{p}}$ and $L_{\mathrm{a}}<L_{\mathrm{p}}$. The modelling approach demonstrated in this work provides a tool for numerical analysis for laser dynamic in multi-mode and multi-cavity QCLs.

\section{REFERENCES}

[1] M. Tonouchi, "Cutting-edge terahertz technology," Nature Photon., vol. 1, pp. $97-105,2007$

[2] P. H. Siegel, "Terahertz technology," IEEE Trans. Microw. Theory Techn., vol. 50, no. 3, pp. 910-928, Mar. 2002

[3] R. Köhler et al., "Terahertz semiconductor-heterostructure laser," Nature, vol. 417, pp. 156-159, May 2002

[4] L. Li et al., "Terahertz quantum cascade lasers with $>1 \mathrm{~W}$ output power," Electron. Lett., vol. 50, pp. 309-311, Feb. 2014

[5] G. Scalari et al., "THz and sub-THz quantum cascade lasers," Lasers Photon. Rev., vol. 3, pp. 45-66, Feb. 2009

[6] C. W. I. Chan, Q. Hu, and J. L. Reno, "Ground state terahertz quantum cascade lasers," Appl. Phys. Lett., vol. 101, p. 151108, Oct. 2012.

[7] M. Wienold et al., "Frequency dependence of the maximum operating temperature for quantum-cascade lasers up to $5.4 \mathrm{THz}$," Appl. Phys. Lett, vol. 107, p. 202101, Nov. 2015.

[8] S. Fathololoumi et al., "Terahertz quantum cascade lasers operating up to $\sim 200 \mathrm{~K}$ with optimized oscillator strength and improved injection tunneling," Opt. Exp., vol. 20, pp. 3866-3876, Feb. 2012.

[9] H.-W. Hübers et al., "High-resolution gas phase spectroscopy with a distributed feedback terahertz quantum cascade laser," Appl. Phys. Lett., vol. 89, p. 061115 , Oct. 2006.

[10] P. Dean et al., "Dual-frequency imaging using an electrically tunable terahertz quantum cascade laser," Opt. Exp., vol. 17, pp. 20631-20641, Oct. 2009.

[11] L. Mahler et al., "Single-mode operation of terahertz quantum cascade lasers with distributed feedback resonators," Appl. Phys. Lett. vol. 84 pp. 5446-5448, Jun. 2004.
[12] O. Demichel et al., "Surface plasmon photonic structures in terahertz quantum cascade lasers," Opt. Exp., vol. 14, pp. 5335-5345, Jun. 2006.

[13] A. W. M. Lee, B. S. Williams, S. Kumar, Q. Hu, and J. L. Reno, "Tunable terahertz quantum cascade lasers with external gratings," Opt. Lett., vol. 35, pp. 910-912, Apr. 2010.

[14] N. Han et al., "Broadband all-electronically tunable MEMS terahertz quantum cascade lasers," Opt. Lett., vol. 39, pp. 3480-3483, Jun. 2014.

[15] S. P. Khanna, M. Salih, P. Dean, A. G. Davies, and E. H. Linfield, "Electrically tunable terahertz quantum-cascade laser with a heterogeneous active region," Appl. Phys. Lett., vol. 95, p. 181101, Oct. 2009.

[16] M. Giehler, H. Kostial, R. Hey, and H. T. Grahn, "Suppression of longitudinal modes in two-sectioned, coupled-cavity $\mathrm{GaAs} /(\mathrm{Al}, \mathrm{Ga})$ as terahertz quantum-cascade lasers," Appl. Phys. Lett., vol. 91, p. 161102, Oct. 2007.

[17] H. Li et al., "Coupled-cavity terahertz quantum cascade lasers for single mode operation," Appl. Phys. Lett., vol. 104, p. 241102, Jun. 2014.

[18] I. Kundu et al., "Discrete Vernier tuning in terahertz quantum cascade lasers using coupled cavities," Opt. Exp., vol. 22, p. 16595, Jun. 2014.

[19] G. Agnew et al., "Efficient prediction of terahertz quantum cascade laser dynamics from steady-state simulations," Appl. Phys. Lett., vol. 106, p. 161105 , Apr. 2015.

[20] L. A. Coldren and T. L. Koch, "Analysis and design of coupled-cavity lasers-Part I: Threshold gain analysis and design guidelines," IEEE J. Quantum Electron., vol. QE-20, no. 6, pp. 659-670, Jun. 1984.

[21] L. A. Coldren and T. L. Koch, "Analysis and design of coupled-cavity lasers-Part II: Transient analysis," IEEE J. Quantum Electron., vol. QE20, no. 6, pp. 671-682, Jun. 1984.

[22] L. A. Coldren, S. W. Corzine, and M. L. Mašanović, Diode Lasers and Photonic Integrated Circuits, 2nd ed. New York, NY, USA: Wiley, 2012, ch. 3 .

[23] S. Barbieri et al., "2.9 THz quantum cascade lasers operating up to 70 K in continuous wave," Appl. Phys. Lett., vol. 85, pp. 1674-1676, Sept. 2004.

[24] V. D. Jovanovic et al., "Influence of doping density on electron dynamics in GaAs/AlGaAs quantum cascade lasers," J. Appl. Phys., vol. 99, p. 103106, May 2006.

[25] D. Indjin, P. Harrison, R. W. Kelsall, and Z. Ikonic, "Self-consistent scattering model of carrier dynamics in GaAs-AlGaAs terahertz quantumcascade lasers," IEEE Photon. Technol. Lett., vol. 15, no. 1, pp. 15-17, Jan. 2003.

[26] P. Harrison and A. Valavanis, Quantum Wells, Wires and Dots: Theoretical and Computational Physics of Semiconductor Nanostructures, 4th ed. New York, NY, USA: Wiley, 2016, ch. 13.

[27] R. Rungsawang et al., "Gain enhancement in a terahertz quantum cascade laser with parylene antireflection coatings," Appl. Phys. Lett., vol. 98, p. 101102, Mar. 2011.

[28] P. Greck, S. Birner, B. Huber, and P. Bogl, "Efficient method for the calculation of dissipative quantum transport in quantum cascade lasers," Opt. Exp., vol. 23, pp. 6587-6600, Jan. 2015.

[29] T. Hagelschuer et al., "High-spectral resolution terahertz imaging with a quantum-cascade laser," Opt. Exp., vol. 24, pp. 13839-13849, Jun. 2016.

[30] M. Wienold et al., "Real-time terahertz imaging through self-mixing in a quantum-cascade laser," Appl. Phys. Lett., vol. 109, p. 011102, Jun. 2016.

[31] T. Hagelschuer et al., "Terahertz gas spectroscopy through self-mixing in a quantum-cascade laser," Appl. Phys. Lett., vol. 109, p. 191101, Oct. 2016.

[32] I. Kundu et al., "Quasi-continuous frequency tunable terahertz quantum cascade lasers with coupled cavity and integrated photonic lattice," Opt. Exp., vol. 25, pp. 486-496, Jan. 2017.

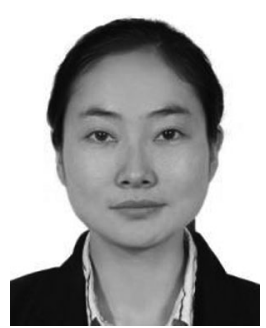

Xiaoqiong Qi was born in Gansu, China, in 1982. She received the B.S. and Ph.D. degrees from Lanzhou University, Lanzhou, China, in 2003 and 2009, respectively. She conducted research with the University of California, Los Angeles, USA, from 2007 to 2009, under a joint-training Ph.D. program. She joined the State Key Laboratory on Integrated Optoelectronics, Institute of Semiconductors, Chinese Academy of Sciences, Beijing, China, in 2010, and became an Associate Professor in 2011. Since 2015, she has been a Postdoctoral Research Fellow with the School of IT and Electrical Engineering, The University of Queensland, Brisbane, Qld., Australia. Her current research interests include laser dynamics in semiconductor lasers, terahertz quantum cascade lasers modeling, terahertz sensing, and microwave photonics. 


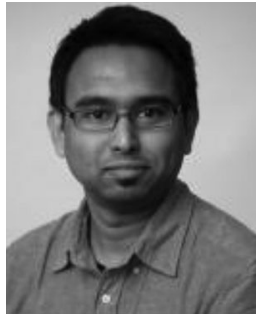

Iman Kundu was born in Kolkata, India, in 1984. He received the B.Tech. degree in electronics and communication engineering from West Bengal University of Technology, Kolkata, in 2007, and the M.Sc. (Eng.) degree in nanotechnology and advanced electronic devices and the Ph.D. degree in electronic and electrical engineering from the University of Leeds, Leeds, U.K., in 2010 and 2014, respectively.

From 2008 to 2009, he was an Assistant Systems Engineer with Tata Consultancy Services, India, working on software development. Since 2014, he has been a Postdoctoral Research Fellow with the University of Leeds. His research interests include fabrication and development of novel waveguides for THz QCLs, laser dynamic in coupled waveguides, fabrication of microstructures on QCL waveguides, and $\mathrm{THz}$ photonics-in-a-chip technology. He received the FW Carter prize in 2015 for the best Ph.D. thesis within the School of Electronic and Electrical Engineering, University of Leeds.

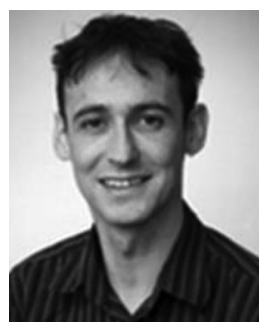

Paul Dean received the M.Phys. (Hons.) degree in physics and the $\mathrm{Ph} . \mathrm{D}$. degree in laser physics from the University of Manchester, Manchester, U.K., in 2001 and 2005, respectively. In 2005, he became a Postdoctoral Research Associate with the Institute of Microwaves and Photonics, School of Electronic and Electrical Engineering, University of Leeds, Leeds, U.K., and a University Research Fellow in 2011. His research interests include terahertz optoelectronics, quantum cascade lasers, and terahertz imaging techniques.

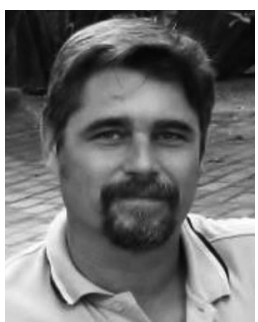

Gary Agnew (M'93) received the B.Sc. and M.Sc. degrees in electrical engineering from the University of the Witwatersrand, Johannesburg, South Africa, in 1985 and 1990, respectively. He is currently working toward the Ph.D. degree at The University of Queensland, Brisbane, Qld., Australia. He has held a variety of research positions in the instrumentation industry, working on microwave, photonic, and nucleonic sensor technology. His research interests include modeling terahertz quantum cascade lasers.

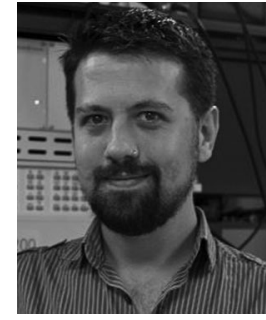

Alexander Valavanis received the M.Eng. (Hons.) degree in electronic engineering from the University of York, York, U.K., in 2004, and the Ph.D. degree in electronic and electrical engineering from the University of Leeds, Leeds, U.K., in 2009.

From 2004 to 2005, he was an Instrumentation Engineer with STFC Daresbury Laboratories, Warrington, U.K. He is currently a University Academic Fellow with the University of Leeds. His research interests include terahertz instrumentation, quantum cascade lasers, silicon photonics, and computational methods for quantum electronics. He is a Member of the Institution of Engineering and Technology.
Andrew T. Grier received the B.Sc. (Hons.) degree in physics from the University of St. Andrews, St Andrews, U.K., in 2009, and the M.Sc. and Ph.D. degrees in electronic and electrical engineering from the University of Leeds, Leeds, U.K., in 2010 and 2015, respectively. He is currently a Senior Research and Development Engineer with Seagate Technology, Londonderry, U.K. His research interests include computational modeling of carrier transport in semiconductor quantum electronics and stochastic micromagnetic modeling of advanced magnetic recording devices.

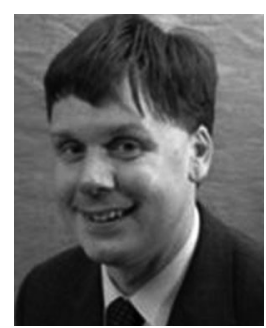

Edmund H. Linfield received the B.A. (Hons.) degree in physics and the M.A. degree from the University of Cambridge, Cambridge, U.K., in 1986 and 1991, respectively, and the Ph.D. degree in semiconductor physics from the Cavendish Laboratory, University of Cambridge, in 1991

He continued his research as a Postdoctoral Researcher Associate with the Cavendish Laboratory, University of Cambridge, until 1997, when he was appointed as an Assistant Director of Research. He also became a Fellow of Gonville and Caius College, Cambridge. In 2004, he moved to the University of Leeds, Leeds, U.K., to take up the Chair of Terahertz Electronics. He is currently also the Director of Research in the School of Electronic and Electrical Engineering, and was awarded a "Dream Fellowship" from the Engineering and Physical Sciences Research Council in 2011. His research interests include semiconductor growth and device fabrication, terahertz-frequency optics and electronics, and nanotechnology.

Prof. Linfield received the IOP Faraday Medal and Prize, jointly with Prof. Davies, in 2014 and a Wolfson Research Merit award from the Royal Society in 2015 .

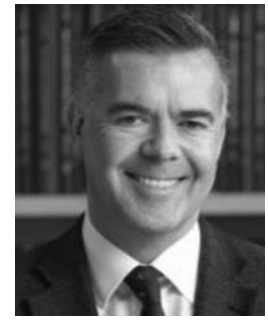

Alexander Giles Davies received the B.Sc. (Hons.) degree in chemical physics from the University of Bristol, Bristol, U.K., in 1987, and the Ph.D. degree from the University of Cambridge, Cambridge, U.K., in 1991.

In 1991, he joined the University of New South Wales, Sydney, Australia, supported by an Australian Research Council Fellowship, before returning to the Cavendish Laboratory, University of Cambridge, in 1995, as a Royal Society University Research Fellow, and subsequently, a Trevelyan Fellow of Selwyn College, Cambridge. Since 2002, he has been with the School of Electronic and Electrical Engineering, University of Leeds, Leeds, U.K., as a Professor of electronic and photonic engineering, and is currently also the Pro-Dean for Research and Innovation in the Faculty of Engineering. His research interests include optical and electronic properties of semiconductor devices, terahertz frequency electronics and photonics, and the exploitation of biological properties for nanostructure engineering. ests include interface of probability theory, computer simulation, and mathematical optimization with biological and other scientific, engineering, and finance disciplines, including within laser feedback interferometry.
Thomas Taimre received the B.Sc. degree in mathfrom The University of Queensland, Brisbane, Qld., is currently a Lecturer of mathematics and statistics with The University of Queensland. He coauthor of the Handbook of Monte Carlo Methods (Wiley, 2011), which provides a hands-on guide to the theory, algorithms, techniques, and applications of Monte-Carlo methods. His current research inter- 


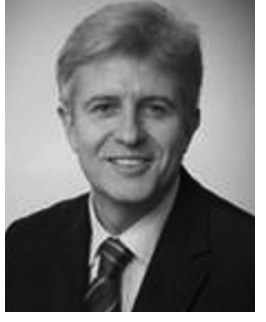

Dragan Indjin received the B.S., M.S., and Ph.D. degrees in electrical engineering from the University of Belgrade, Belgrade, Yugoslavia.

He joined the Faculty of Electrical Engineering, University of Belgrade, in 1989, where he later became an Associate Professor. Since 2001, he has been with the Institute of Microwaves and Photonics, School of Electronic and Electrical Engineering, University of Leeds, Leeds, U.K., where he is currently a Reader (Associate Professor) in Optoelectronics and Nanoscale Electronics. He is currently focused on applications of quantum-cascade lasers and interband cascade lasers for sensing and imaging applications. His research interests include electronic structures, optical and transport properties, optimization and design of quantum wells, superlattices, quantum-cascade lasers, and quantum-well infrared photodetectors from near- to far-infrared and terahertz spectral ranges. He received the prestigious Academic Fellowship from the Institute of Microwaves and Photonics, School of Electronic and Electrical Engineering, University of Leeds, in 2005. $\mathrm{He}$ is currently a Coordinator of major international projects on infrared and terahertz imaging and sensing for medical and security applications.

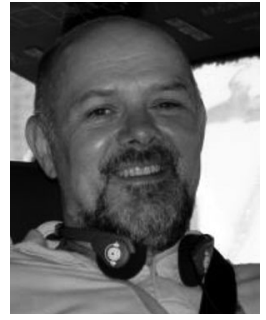

Aleksandar D. Rakić (M'93-SM'10) is currently a Professor with the School of IT and Electrical Engineering, The University of Queensland, Brisbane, Qld., Australia. He leads the Microwave, Photonics, and Communications Group, The University of Queensland, focusing on the development of technologies for sensing and imaging across the electromagnetic spectrum including microwave, terahertz wave, and optical systems. Over the past ten years, his group pioneered the development of several world's first laser-feedback interferometric sensors including systems based on monolithic vertical-cavity surface-emitting laser arrays (VCSELs), blue-green lasers, terahertz quantum cascade lasers, and midinfrared interband cascade lasers. His current research interests include development of sensing and imaging systems, exploiting the $\mathrm{THz}$ spectrum for applications from security and defense to in-vivo biomedical imaging. His other principal contributions relate to the design and characterization of surface-emitting optoelectronic devices (VCSELs and light emitting diodes). He was appointed as an Invited Professor at the University of Toulouse in 2007-2012, and a Visiting Professor at the University of Leeds in 2012. He served as the General Chair of the "Conference on Optoelectronic and Microelectronic Materials and Devices" in 2004, the Co-Chair of the "Symposium on Molecular and Organic Electronics and Organic Displays" within the International Conference on Nanoscience and Nanotechnology in 2006, the Chair of the "Symposium on Compound Semiconductor Materials and Devices" within the International Conference on Electronic Materials in 2008, and as the Chair of the IEEE AP/MTT Queensland Chapter. 